\title{
A Case of Acquired Hemophilia A with Maxillary Osteitis
}

\author{
Omar EL Graoui, Saadia Faez, Bouchra Oukkache \\ From the Department of Hematology Laboratory, Ibn Rushd Teaching Hospital, Casablanca University, Morrocoo
}

\footnotetext{
Citation: El Graoui O, Faez S, Oukkache B. A Case of Acquired Hemophilia A with Maxillary Osteitis. Maced J Med Sci. 2012 Dec 15; 5(4):434436. http://dx.doi.org/10.3889/MJMS.19575773.2012.0241.

Key words: Acquired hemophilia A; infection; Factor VIII inhibitor.

Correspondence: Dr. Oukkache Bouchra Louai. Teaching Hospital CHI Ibn Rochd, Hematology Laboratory, Casablanca 20200, Morocco. E-Mail: bouchraoukkache@gmail.com

Received: 24-Jan-2012; Revised: 02-May-2012; Accepted: 29-May-2012; Online first: 10-Oct-2012

Copyright: @ 2012 El Graoui O. This is an openaccess article distributed under the terms of the Creative Commons Attribution License, which permits unrestricted use, distribution, and reproduction in any medium, provided the origina author and source are credited.

Competing Interests: The author have declared that no competing interests exist.
}

\begin{abstract}
Acquired hemophilia $\mathrm{A}(\mathrm{AHA})$, is a rare bleeding disorder with an incidence of approximately 1 per million/year. The disease is caused by the development of autoantibodies directed against clotting factor VIII and associated with an increased morbidity and mortality. In 50\% of cases, FVIII autoantibodies occur in patients lacking any relevant concomitant disease; the remaining cases may be associated with postpartum period, autoimmune diseases, underlying hematologic or solid cancers, and infections.
\end{abstract}

We report the case of a 32-years-old woman, admitted in hemorrhagic shock, following a non-reversible bleeding after dental extraction. The Laboratory tests prolonged activated prothrombin thromboplastin time (APTT at 54s), and normal Prothombine time (PT at 75\%). The mixing test revealed the presence of a circulating anticoagulant with a Rosner index at 15. Factors specific to the intrinsic pathway determined that the patient had a factor VIII deficiency. Acquired hemophilia was diagnosed.

At the local level, CT scan of the face objectified a right maxillary osteitis without sinus damage. The patient received transfusion of Red Blood Cells units and FFP (fresh frozen plasma) concentrates.but kept bleeding until an antibiotic treatment and corticosteroids $(60 \mathrm{mg} / \mathrm{d})$ were added.

\section{Introduction}

First described on 1940, Acquired hemophilia A $(\mathrm{AHA})$, is a rare bleeding disorder but is a serious cause of haemorrhage with an incidence of approximately 1 per million/year [1, 2].The disease is caused by the development of autoantibodies directed against clotting factor VIII and associated with an increased morbidity and mortality.

Acquired haemophilia is associated with autoimmune diseases, malignancy, dermatological disorders, drug interactions, postpartum period and infection $[3,5]$.

Clinical features are hemorrhages into the skin, muscles, or soft tissues and mucous membranes [5]. Hemorrhagic complications are common; they are fatal in $8 \%-22 \%$ of the patients [2], with most deaths occurring in the first few weeks after presentation.

The diagnosis of acquired hemophilia is based on a prolonged activated partial thromboplastin time, which may be not corrected initially by mixing the patient's 
El Graoui et al. A Case of Acquired Hemophilia A with Maxillary Osteitis

plasma with an equal volume of normal plasma.

Therapy for acquired hemophilia focuses on 2 goals: control of acute bleeding and chronic immunosuppressive therapy to prevent further development of factor VIII inhibitors.

We report a case of acquired hemophilia, occurring in a patient with dental infection.

\section{Case description}

32-years-old women, mother of two children (7 years and 9 months) were admitted in hemorrhagic shock, following a non-reversible bleeding after dental extraction. No other bleeding symptoms were reported, and neither history of liver disease, nor personal or family history of bleeding or clotting disorders was associated.

Her past medical history included heavy bleeding during the birth of the $2^{\text {nd }}$ child non explored. She was not taking anticoagulants or non steroidal anti-inflammatory drugs.

Her clinical examination showed palor, fever at $38.8^{\circ} \mathrm{C}$, accelerated pulse, blood pressure at 90/60 $\mathrm{mmHg}$, a massive buccal hemorrage, with no bruises nor other bleeding symptoms.

The Laboratory tests objectified an $\mathrm{Hb}$ at $7 \mathrm{~g} / \mathrm{dl}$, the platelet count and the white blood cells count were normal; with prolonged activated prothrombin thromboplastin time (APTT at 54s) (Normal range: $35 \mathrm{~s}$ $\pm 5 \mathrm{~s}$ ), and normal Prothombine time (PT at 75\%) . the mixing test revealed the presence of a circulating anticoagulant with a Rosner index at 15 ( A 1:1 mixing assay initially showed a corrected activated partial thromboplastin time of 41 seconds; however, a timedelayed 1:1 mix could not correct the thromboplastin time, which suggested that clotting factor inhibitors were present in the patient's blood. Because only the activated partial thromboplastin time was affected, we assayed for clotting factors specific to the intrinsic pathway and determined that the patient had a factor VIII deficiency which rate was 36\%(Normal range: $70 \%$ - 140). The quantification of Inhibitors was made by the Bethesda assay (titre $5.10 \mathrm{U} / \mathrm{ml}$ [normal 0.5-1.5] U/mL) .Von Willebrand antigen (WF: Ag) rate was at $73 \%$.

Test results of rheumatoid factor, antinuclear antibody and anticardiolipin IgG were all normal or negative. We made a diagnosis of acquired haemophilia.
At the local level, CT scan of the face objectified a right maxillary osteitis without sinus damage.

The patient received at admission transfusion of Red Blood Cells units and FFP (fresh frozen plasma) concentrates, with local fibrin glue, but she kept bleeding. After diagnosis of AHA, the antibiotic treatment and the corticosteroids (60 mg/j) were added.

After one week from the start of the treatment, she stopped bleeding, with good improvement of her physical condition. Hemostasis went to normal, and inhibitors disappeared.

\section{Discussion}

Acquired hemophilia $\mathrm{A}(\mathrm{AHA})$ is an uncommon but life-threatening hemorrhagic disorder caused by the presence of autoantibodies directed against the coagulation factor VIII (FVIII) [2]. Epidemiological, clinical, and biological characteristics are distinct from those of classic hemophilia $A$, as well as from the unusual situation of patients with hemophilia A who develop alloantibodies against factor VIII after receiving infusions of factor VIII concentrate [3]. The age distribution of autoantibodies is typically biphasic with a small peak between 20 and 30 years due to post-partum; and a major peak in patients aged 68-80 years. The incidence in males and females is similar except in 20-40 year olds where the effect of pregnancy results in a preponderance of females [2]. In $50 \%$ of cases, FVIII autoantibodies occur in patients lacking any relevant concomitant disease; the remaining cases may be associated with postpartum period, autoimmune diseases (systemic lupus erythematosus, rheumatoid arthritis, thyroid disorders and so on), underlying hematologic or solid cancers, infections like maxillary osteitis in our patient, vaccination or use of medications [4]. In malignancy associated AHA, there is no clear relationship between the type and extent of the tumor and the occurrence or severity of AHA [1].

Most patients with FVIII autoantibodies have hemorrhages into the skin, muscles, or soft tissues and mucous membranes (e.g., epistaxis, gastrointestinal and urological bleeds, retroperitoneal hematomas, and postpartum bleeding), whereas hemarthrosis, a typical feature of congenital FVIII deficiency, are uncommon [5]. The hemorrhages are often life threatening, and the disease may manifest more dramatically by excessive bleeding following trauma or surgery or by cerebral hemorrhage [6]. Life-threatening hemorrhage occurs in about $8-22 \%$ of cases [2] while mild bleeding, requiring 
no treatment, is seen in about $30 \%$ of cases [1]. In our patient, it was buccal hemorrhage, causing hemorrhagic shock.

The diagnosis of AHA is based on demonstrating an isolated prolongation of activated prothrombin thromboplastin time (APTT), not corrected by incubating for $2 \mathrm{~h}$ at $37^{\circ} \mathrm{C}$ patient's plasma with equal volumes of normal plasma (mixing study), associated with a reduced FVIII level and evidence of FVIII inhibitor activity in a patient with no previous personal or family history of bleeding [2]. In our patient, there was past medical history of bleeding,but laboratory tests confirmed the diagnosis of $\mathrm{AHA}$, with positive mixing study, and isolated low rate of factor VIII. Factor VIII inhibitor can be titrated using the Bethesda assay, which measures residual FVIII activity after incubation of patient plasma with normal plasma for $2 \mathrm{~h}$ at $37^{\circ} \mathrm{C}$.According to the Bethesda assay, inhibitors are classified at low or high [2]. However, before confirming the presence of FVIII inhibitor, the possible coexistence of heparin or lupus anticoagulant should be ruled out. The presence of heparin is suggested by a prolonged thrombin time with normal reptilase time, while a similar APTT prolongation of the mixture patient/ normal plasma at time 0 and after incubation is indicative for lupus anticoagulant [2].

Treatment for acute bleeding episodes is directed towards the temporary restoration of blood clotting to normal.Infusions of FFP are generally useless because their small content of factor VIII is rapidly inactivated by circulating antibodies. Larger than normal and sometimes massive doses of human factor VIII concentrate, antifibrinolytics and DDAVP, may be effective, particularly if the anti-VIII titre is low.Plasma exchange is occasionnaly helpful.But the main treatment relies on bypassing agents. The two licensed treatment products are rFVIIa, (NovoSeven $\AA$ ) and FEIBA $\AA[1]$. A retrospective analysis by Hay et al. [7] showed that $100 \%$ response in patients with AHA when rF VIIa was used as a first-line treatment and approximately about $75 \%$ response was observed when it was used at a late stage.[1].Another retrospective study of 34 patients with AHA showed an overall complete response rate of $86 \%$ with a dosing regimen of FEIBA at $75 \mathrm{U} / \mathrm{kg}$ every 8-12 h with a median number of 10 doses to control severe bleeding [1]. The use of bypassing agents in AHA is associated with thrombotic events [1].

The second approach of treatment is eradication of inhibitors. The two most common regimens are corticosteroids alone, that worked with our patient; or corticosteroids combined with cyclophosphamide [1] Infusion of intravenous immunoglobulin (IVIG) has also been used as therapy. Immunoadsorption using staphylococcal protein A to remove the autoantibodies to FVIII [1] may be useful in bleeding or presurgical patients with high titer inhibitors who have failed to respond to bypassing agents. Recently, rituximab has emerged as a promising new agent for the eradication of inhibitors in patients with acquired hemophilia [1].

\section{Conclusion}

Acquired hemophilia $A$ is a rare and lifethreatening condition.An underlying disease is common, and should be searched for. Patient's prognosis has been improved, due to the progresses in the understanding of the biology of the disease and the development of novel treatment protocols.

\section{References}

1. Shetty S, Bhave M, Ghosh K. Acquired hemophilia A: Diagnosis, aetiology, clinical spectrum and treatment options. Autoimmunity Reviews. 2011;10:311-316.

2. Franchini M, Targher G, Montagnana M, Lippi G. Laboratory, clinical and therapeutic aspects of acquired hemophilia A. Clinica Chimica Acta. 2008;395:14-18.

3. Bossi P, Cabane J, Ninet J. Acquired Hemophilia Due to Factor VIII Inhibitors in 34 Patients. Am J Med. 1998;105.

4. Moulis G, Pugnet G, Bagheri $\mathrm{H}$, et al. Acquired factor VIII hemophilia following influenza vaccination. Eur $\mathrm{J}$ Clin Pharmacol. 2010;66:1069-70.

5. Collins P, Hirsch S, Baglin TP, et al. Acquired hemophilia A in the United Kingdom: a 2-year national surveillance study by the United Kingdom Haemophilia Centre Doctors' Organisation. Blood. 2007;109:1870-7.

6. Franchini M, Gandini G, Di Paolantonio T, Mariani G. Acquired hemophilia A: a concise review. Am J Hematol. 2005;80:55-63.

7. Hay CR, Negrier C, Ludlam CA. The treatment of bleeding in acquired hemophiliawith recombinant factor VIIa: a multicenter study. Thromb Haemost. 1997;78:1463-7. 